\title{
Personalized Health Informatics
}

\author{
Antoine Geissbuhler, President of IMIA \\ Geneva University Hospitals, Switzerland
}

It is indeed exciting to work in a field where challenges evolve rapidly and continuously. This is the privilege of disciplines, such as ours, that lie at the intersection of many scientific and societal domains. As physical and digital aspects of our lives mingle increasingly, the prospect of informatics making a real difference to health outcomes and healthcare processes becomes ever more realistic, but also generates high expectations. Our community is therefore challenged to tackle some of the most difficult health-related societal issues such as the provision of equitable access to quality healthcare, the support for more efficient biomedical research, and the empowerment of citizens to better manage their health.

For two decades, the IMIA Yearbook has been attempting to demonstrate how our health informatics community is advancing in dealing with these challenges, by highlighting excellent stateof-the -art research, by providing surveys and opinion papers about current developments, and by sharing information about our global, regional, and local activities. The first decade of the Yearbook was directed by its two co-founders, Jan van Bemmel and Alexa McCray. The second decade was co-edited by Reinhold Haux, Casimir Kulikowski and I. The time has come to thank the longest-serving co-editor of the Yearbook ever, Casimir Kulikowski, who will be retiring this year from this position. For twelve years, he brought creativity, out-of-thebox thinking, an extensive knowledge of our field and of its history, a special care for details and accuracy, and, most of all, lots of humor and an excellent sense of conviviality for such teamwork. The third decade of the Yearbook will start under the leadership of a new team, with Marie-Christine Jaulent and Brigitte
Séroussi as co-editors, and Christoph Lehmann, as incoming IMIA vice-president for services. They will undoubtedly bring fresh ideas in order to best inform and serve our IMIA community as well as wider audiences.

IMIA's role as a global convener, facilitator of creative thinking, and promoter of best practices becomes more apparent as biomedical informatics gets embedded even more closely into healthcare systems and biomedical research. Several current IMIA-led initiatives illustrate how our strategic plan is now being implemented by Peter Murray, IMIA's CEO, and his excellent team, with the guidance of IMIA's board and general assembly.

First, the open-access publication of a special issue of the WHO Bulletin, establishing the current evidence base for the impact of eHealth, or relative lack thereof, and the need for concerted actions to better measure the added value of health informatics tools and systems [1]. This effort was supported by funding from PEPFAR, IDRC and the Rockefeller Foundation. More than seventy contributions were submitted, but only a fraction could be published. Others will be considered for another special issue on this important theme, to be published in the International Journal of Medical Informatics, an official journal of IMIA. Actions will follow from these analyses, including work on policies and the development of tools to help implement them effectively, such as the definition of a set of consistent indicators to measure the impact of eHealth interventions, and a database of eHealth related trials. Such activities are planned as part of a strengthened collaboration plan between WHO and IMIA. Other partnerships will also need to be developed, in particular in the field of health information standards.
Second, the hosting of the $2012 \mathrm{Eu}-$ ropean Summit on Trustworthy Reuse of Health Data [2], which, in collaboration with the European Federation of Medical Informatics, brought together government representatives, academia and the industry to jointly work to define and agree on key issues and possible solutions to facilitate research on the uses of health and healthcare record data. Subsequent activities include various publications targeted at several different key audiences, as well as work on policies, and, in particular, a framework that could help organize large-scale sharing of data and its meaningful and ethical reuse for research and healthcare service development.

Third, the validation phase of an IMIA-led accreditation process for health informatics educational programs, designed by Arie Hasman, IMIA's vicepresident for special affairs. The goal of this project is to improve sharing of best practices in health informatics education, through a solid process supported by experts from the IMIA community. Once validated on several test sites, this accreditation process will be made available more widely.

These three examples of recent activities also highlight how each of us can, personally, using IMIA's expertise and collaborative platform, contribute to help address some of the challenging issues our field faces, and pave the way for innovative solutions. This is yet another way of looking at "personalized health informatics".

\section{References}

1. http://www.who.int/bulletin/volumes/90/5/en/ index.html, last accessed on July $1^{\text {st }}, 2012$

2. http://euhealthdata2012.imia.info/, last accessed on July $1^{\text {st }}, 2012$ 